\title{
Delayed kidney injury following coronary angiography
}

\author{
FENG WANG $^{1 *}$, CHENG PENG $^{2 *}$, GUANGYUAN ZHANG $^{3 *}$, QING ZHAO $^{4}$, \\ CHANGYOU XUAN ${ }^{1}$, MENG WEI $^{4}$ and NIANSONG WANG ${ }^{1}$
}

\begin{abstract}
Departments of ${ }^{1}$ Nephrology and Rheumatology, and ${ }^{2}$ Cardiothoracic Surgery, Affiliated Sixth People's Hospital, Shanghai Jiao Tong University, Shanghai 200233; ${ }^{3}$ Department of Urology, Shanghai First Hospital, Shanghai Jiao Tong University School of Medicine, Shanghai 200025; ${ }^{4}$ Department of Cardiology, Affiliated Sixth People's Hospital, Shanghai Jiao Tong University, Shanghai 200233, P.R. China
\end{abstract}

Received February 4, 2015; Accepted April 12, 2016

DOI: $10.3892 / \mathrm{etm} .2016 .3315$

\begin{abstract}
It is occasionally observed that patients without contrast-induced nephropathy (CIN) develop kidney injury within 1-6 months after coronary angiography (CAG), termed delayed CIN or delayed kidney injury (DKI) following CAG. The present study aimed to investigate the associated risk factors of delayed CIN and its possible pathogenesis. Subjects with CAG or coronary stenting from January 2008 to December 2009 were studied. A retrospective survey on DKI after CAG was conducted and the risk factors were analyzed. There were 436 cases receiving CAG with complete medical records enrolled in the present cohort, in which the DKI incidence was $7.1 \%$ (31/436). Patients with DKI after CAG exhibited lower hemoglobin $(121.2 \pm 17.3$ vs. $133.8 \pm 18.6 \mathrm{~g} / \mathrm{l})$, estimated glomerular filtration rate (eGFR; $66.4 \pm 30.2$ vs. $71.9 \pm 28.6 \mathrm{ml} / \mathrm{min}$ ), higher serum creatinine $(110.9 \pm 43.2$ vs. $91.7 \pm 37.6 \mu \mathrm{mol} / \mathrm{l})$, higher rate of heart failure (22.6 vs. $5.4 \%$ ) and $300 \mathrm{mg}$ aspirin therapy ( 29 vs. $5.7 \%$ ) compared with non-DKI patients (all $\mathrm{P}<0.05$ ). However, no differences were observed in morbidities of diabetes, hypertension, hyperlipidemia and proteinuria, or in the treatments with angiotensin converting enzyme
\end{abstract}

Correspondence to: Dr Feng Wang or Dr Niansong Wang, Department of Nephrology and Rheumatology, Affiliated Sixth People's Hospital, Shanghai Jiao Tong University, 600 Yishan Road, Shanghai 200233, P.R. China

E-mail: zyzwq1030@gmail.com

E-mail: wangniansong2008@163.com

*Contributed equally

Abbreviations: CIN, contrast-induced nephropathy; CAG, coronary angiography; DKI, delayed kidney injury; eGFR, estimated glomerular filtration rate; NSAIDs, non-steroid anti-inflammatory drugs; ACEI/ARB, angiotensin converting enzyme inhibitors/angiotensin II receptor blockers; CKD, chronic kidney disease

Key words: contrast-induced nephropathy, acute kidney injury, coronary angiography, aspirin, heart failure
(ACE) inhibitors/angiotensin II receptor-1 blockers (ARBs), diuretics, statins and other anti-platelets between the two groups $(\mathrm{P}>0.05)$. Logistic regression revealed that anemia, heart failure and $300 \mathrm{mg}$ aspirin intake were risk factors of DKI $(\mathrm{P}<0.05)$, while the contrast level, isotonic contrast, diabetes, ACE inhibitors/ARBs, eGFR and other factors were not associated with DKI $(\mathrm{P}>0.05)$. Heart dysfunction and $300 \mathrm{mg}$ aspirin therapy may contribute to DKI after CAG, and iodinated contrast media administration is not a risk factor.

\section{Introduction}

Contrast-induced nephropathy (CIN) is an acute kidney injury following administration of iodinated contrast media, and is currently the third most common type of hospital-acquired renal failure (1). CIN often appears in patients who underwent coronary angiography (CAG), and may result in renal function deterioration and in certain cases, death. CIN following CAG often causes long-term decline in renal function (2). CIN is defined as an increase in serum creatinine concentration $>26.5 \mu \mathrm{mol} / 1$ or $>25 \%$ of its baseline creatinine level within 3 days after contrast medium administration (3). Typically after a peak value of serum creatinine within the fifth day, in which granular casts and moderate proteinuria may appear, serum creatinine levels return to the baseline level within 7-10 days (4).

However, it has also been observed at Shanghai Jiao Tong University Affiliated Sixth People's Hospital (Shanghai, China) that certain patients that do not exhibit CIN develop irreversible deterioration of renal function within 1-6 months following CAG. This is informally called 'delayed contrast-induced nephropathy' or 'delayed kidney injury (DKI) after CAG', and it has been hypothesized by several cardiologists at the Shanghai Jiao Tong University Affiliated Sixth People's Hospital to be due to iodinated contrast media administration. However, it is unknown whether delayed CIN really exists, or whether it is associated with CAG or induced by iodinated contrast media.

In the present study, it was hypothesized that DKI after CAG may be caused by numerous factors, but that contrast did not contribute. In order to investigate the pathogenesis of DKI, a retrospective study was conducted in patients receiving CAG and coronary stenting to investigate whether DKI exists and to understand its etiology and mechanism of action. 


\section{Materials and methods}

Subjects. Patients receiving CAG and coronary stenting between January 1, 2008 and December 31, 2009 at the Shanghai Jiao Tong University Affiliated Sixth People's Hospital were enrolled in the present study. The inclusion criteria were as follows: Age, $\geq 18$ years old; receiving CAG and percutaneous coronary intervention. The present study was approved by the Ethics Committee of Shanghai Jiao Tong University Affiliated Sixth People's Hospital and adhered to the Declaration of Helsinki (5). Written informed consent was obtained from all of the participants.

The exclusion criteria were as follows: Diagnosis of CIN; electrophysiological examination and percutaneous transluminal septal myocardial ablation conducted; baseline estimated glomerular filtration rate (eGFR) $<30 \mathrm{ml} / \mathrm{min}$; occurrence of malignant tumors, renal artery stenosis and urological obstruction illnesses; recurrence of myocardial infarction during follow-up; renal toxic medicine intake (except aspirin); prerenal acute kidney injury (AKI); and AKI secondary to primary kidney diseases.

Diagnostic criteria and methods. The CIN diagnostic criteria included the following: Cases with a serum creatinine increase (after CAG) $\geq 25 \%$ within $24-72 \mathrm{~h}$ compared with baseline values; or an absolute increase $>44.2 \mu \mathrm{mol} / 1$ (6). The risk scores were taken into account in accordance with the risk score described by Mehran (7). The CAG-associated DKI diagnostic criteria were as follows: Any serum creatinine value increase $\geq 26.5 \mu \mathrm{mol} / 1$ or $>50 \%$ of the baseline value, 1-6 months following CAG. The baseline creatinine was the serum creatinine level at 1 month after CAG. The eGFR was calculated in accordance with the simplified Cockcroft-Gault (CG) formula as follows: CG-eGFR: Creatinine clearance $=(140$-age) $\mathrm{x}$ weight $\times 0.85$ (if female) $/(72 \times$ serum creatinine) (8). The hospital and follow-up medical records of enrolled patients were collected and the different cases were divided into groups, those cases diagnosed with DKI (the DKI group) and those cases without DKI (the non-DKI group). The Mehran scores of the DKI group were calculated and common clinical characteristics were screened (7).

Statistical analysis. SPSS software, version 13.0 was employed for data analysis. Student's t-test was used to analyze the measurement data while the $\chi^{2}$ test was used for categorical comparison. The risk factors associated with DKI, including age, gender, body weight, hospital stay, heart function grade, hemoglobin, baseline serum creatinine, fasting plasma glucose, eGFR, contrast dosage, isotonic contrast, urine protein, diabetes, angiotensin converting enzyme (ACE) inhibitors/angiotensin II receptor blockers (ARBs), aspirin, diuretics and hydration therapy, were analyzed by logistic regression. $\mathrm{P}<0.05$ was considered to indicate a statistically significant difference.

\section{Results}

Basic information of DKI and non-DKI subjects. A total of 769 patients were receiving CAG in the present study, and there were 333 cases excluded according to the exclusion criteria, including 47 cases with definite CIN and 29 drop-out
Table I. Basic information of patients with DKI

\begin{tabular}{lcc}
\hline Characteristic & $\begin{array}{c}\text { Non-DKI } \\
(\mathrm{n}=405)\end{array}$ & $\begin{array}{c}\text { DKI } \\
(\mathrm{n}=31)\end{array}$ \\
\hline Age, years & $65.3 \pm 11.9$ & $69.1 \pm 12.4^{\mathrm{a}}$ \\
Male, N (\%) & $273(67.4)$ & $22(80.0)$ \\
Proteinuria, N (\%) & & \\
Absent & $247(61.0)$ & $16(51.6)$ \\
Microalbuminuria & $131(32.3)$ & $12(38.7)$ \\
Proteinuria & $27(6.7)$ & $3(9.7)$ \\
Comorbities, N $(\%)$ & & $14(45.2)$ \\
Diabetes mellitus & $122(30.1)$ & $21(67.7)$ \\
Hypertension & $276(68.1)$ & $20(64.5)$ \\
Hyperlipidemia & $319(78.8)$ & $7(22.6)^{\mathrm{a}}$ \\
Heart failure & $22(5.4)$ & $6(19.4)^{\mathrm{a}}$ \\
CVD & $34(8.4)$ & $5(16.1)$ \\
PVD & $33(8.1)$ & \\
\hline
\end{tabular}

Age data are presented as the mean \pm standard deviation. ${ }^{\mathrm{a}} \mathrm{P}<0.05$ vs. Non-DKI. DKI, delayed kidney injury; CVD, cerebrovascular disease; PVD, peripheral vascular disease.

Table II. Biochemical parameters in patients with DKI.

\begin{tabular}{lcc}
\hline Parameter & Non-DKI & DKI \\
\hline White blood cell, $10^{9} / 1$ & $6.4 \pm 1.2$ & $6.6 \pm 1.5$ \\
Hemoglobin, g/l & $133.8 \pm 18.6$ & $121.2 \pm 17.3^{\mathrm{a}}$ \\
Hematocrit, $\%$ & $38.6 \pm 5.4$ & $35.1 \pm 5.3^{\mathrm{a}}$ \\
Alanine transaminase, U/1 & $35.2 \pm 13.6$ & $37.4 \pm 12.9$ \\
Serum creatinine, $\mu \mathrm{mol} / \mathrm{l}$ & $91.7 \pm 37.6$ & $110.9 \pm 43.2^{\mathrm{a}}$ \\
Tc, mmol/1 & $4.6 \pm 1.1$ & $4.7 \pm 1.5$ \\
Low-density lipoprotein, g/l & $3.1 \pm 0.9$ & $3.2 \pm 1.1$ \\
eGFR, ml/min & $71.9 \pm 28.6$ & $66.4 \pm 30.2^{\mathrm{a}}$ \\
\hline
\end{tabular}

${ }^{\mathrm{a}} \mathrm{P}<0.05$ vs. non-DKI. eGFR, estimated glomerular filtration rate; Tc, total cholesterol.

cases. In total, there were 436 valid cases with intact follow-up data enrolled in the present study. In addition, the eGFR levels for all the subjects prior to CAG were $>30 \mathrm{ml} / \mathrm{min}$. The incidence of DKI was $7.1 \%(31 / 436)$ and the average time after CAG that DKI occurred was $16.5 \pm 4.0$ weeks. The percentage of heart failure and cerebrovascular disease were significantly higher in DKI patients than that in the non-DKI group ( $\mathrm{P}=0.009$ and 0.041 , respectively). However, no differences in proteinuria, diabetes, hypertension or hyperlipidemia were observed between the groups (Table I). Furthermore, the cases were divided into the non-DKI and DKI group, and the results presented significant differences in hemoglobin $(\mathrm{Hb})$, hematocrit and serum creatinine levels $(\mathrm{P}=0.039,0.043$ and 0.033 , respectively; Table II), between the two groups but no differences in white blood cell, alanine transaminase and low-density lipoprotein. 
Table III. Medicine intake of the DKI patients during the follow-up.

\begin{tabular}{lcc}
\hline Medicine & $\begin{array}{c}\text { Non-DKI (n, \%) } \\
\mathrm{n}=405\end{array}$ & $\begin{array}{c}\text { DKI (n, \%) } \\
\mathrm{n}=31\end{array}$ \\
\hline ACEI & $181(44.7)$ & $16(51.6)$ \\
ARB & $99(24.4)$ & $5(16.2)$ \\
Diuretics & $241(59.5)$ & $20(64.5)$ \\
Statins & $258(63.7)$ & $22(71.0)$ \\
Anti-platelet drugs & & \\
Asp 100 mg & $15(3.7)$ & $1(3.2)$ \\
Clop & $36(8.9)$ & $1(3.2)$ \\
War & $10(24.7)$ & $1(3.2)$ \\
Asp 100 mg + clop & $243(60.0)$ & $14(51.6)$ \\
Cilostazol + clop & $47(11.6)$ & $3(9.7)$ \\
Asp100 mg + clop + war & $31(7.6)$ & $2(6.5)$ \\
Asp 300 mg + clop & $23(5.7)$ & $9(29)^{\mathrm{a}}$ \\
\hline
\end{tabular}

${ }^{\mathrm{a}} \mathrm{P}<0.01$ vs. Non-DKI. DKI, delayed kidney injury; ACEI, angiotensin converting enzyme inhibitor; ARB, angiotensin receptor blocker; Asp, aspirin; clop, clopidogrel; war, warfarin .

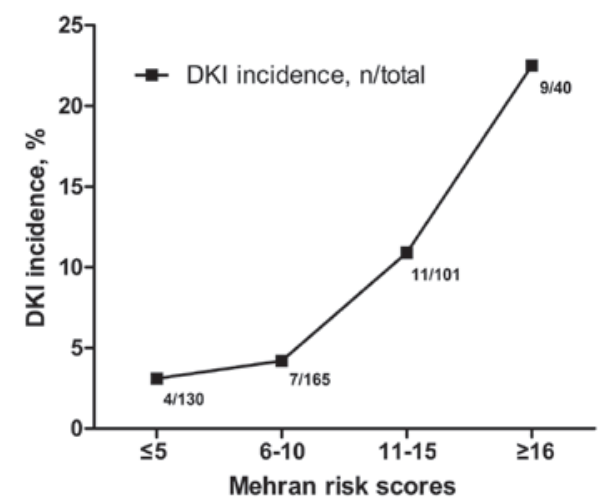

Figure 1. Changes of DKI incidence with Mehran risk scores. The incidence of DKI increased with Mehran risk scores. DKI incidence was 22.5\% (9/40) in patients with scores $\geq 16$. DKI, delayed kidney injury.

DKI incidence increased with Mehran risk scores. The results demonstrated that the incidence rate of DKI increased with Mehran risk scores (Fig. 1), with a DKI incidence of $22.5 \%$ $(9 / 40)$ in patients with scores $\geq 16$.

DKI may be attributed to high-dosage aspirin intake. As presented in Table III, a total of 29\% (9/31) and 5.7\% (23/405) of patients in the DKI and non-DKI groups, respectively, were treated with $300 \mathrm{mg}$ aspirin + clopidogrel following CAG, presenting a significant difference of $\mathrm{P}<0.01$. However, no difference was observed in the number of cases treated with ACE inhibitors/ARBs, diuretics, statins and other anti-platelet drugs (all $\mathrm{P}>0.05$ ).

Multivariate logistic regression analysis was performed to identify the risk factors for DKI following CAG (Table IV). The analyzed factors included age, gender, body weight, hospital stay, heart function grade, hemoglobin, baseline
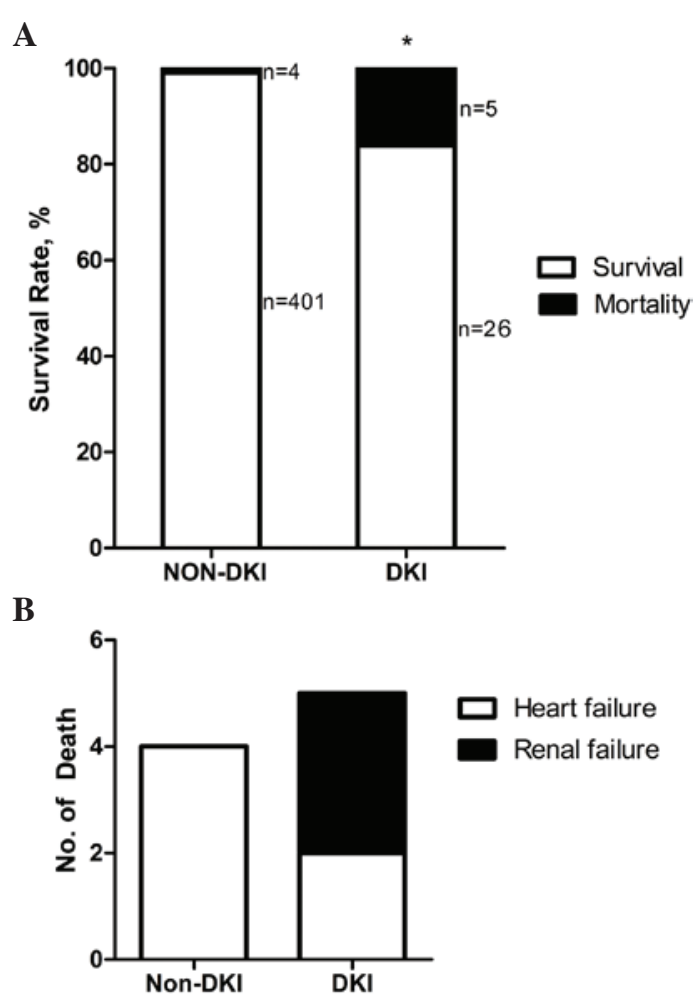

Figure 2. Prognosis of DKI after coronary angiography. (A) In total there were 26 cases with DKI that survived until the follow-up of 6 months, with a mortality rate of $16.1 \%(5 / 31)$. In addition, there were 402 cases without DKI that survived, with a death rate of $1.0 \%(4 / 405) .{ }^{*} \mathrm{P}<0.01$ vs. mortality rate in the non-DKI group. (B) Causes of mortality following DKI included heart and renal failure. DKI, delayed kidney injury.

serum creatinine, fasting plasma glucose, eGFR, contrast dosage, isotonic contrast, urine protein, diabetes, ACE inhibitors/ARBs, aspirin, diuretics and hydration therapy. The results demonstrated that anemia (hemoglobin $<110 \mathrm{~g} / \mathrm{l}$ ), heart failure and $300 \mathrm{mg}$ aspirin intake were risk factors for DKI $(\mathrm{P}<0.05)$, whereas the contrast amount, isotonic contrast, diabetes, ACE inhibitors/ARBs, eGFR and other factors were not associated with DKI $(\mathrm{P}>0.05)$.

Poor prognosis for patients with DKI following CAG compared with non-DKI patients. As presented in Fig. 2A, 26 patients with DKI, out of a total of 31, survived until the follow-up of 6 months; the mortality rate was $16.1 \%$. In addition, there were 402 cases without DKI, out of a total of 405 , that survived, and the mortality rate in this group was $1.0 \%(\mathrm{P}<0.01)$. Causes of mortality following DKI included heart and renal failure (Fig. 2B).

\section{Discussion}

Patients with coronary heart disease often present a high-risk of CIN, therefore studies on the prevention of CIN following CAG is of note (9-13). By contrast, coronary artery-associated illnesses such as acute coronary syndrome, myocardial infarction and ischemic cardiomyopathy may lead to heart failure and decreased renal blood flow and kidney injury $(14,15)$. The present study hypothesized that DKI following CAG may not be a result of contrast itself, but other associated factors. In 
Table IV. Results of univariate and multivariate analysis for DKI.

\begin{tabular}{|c|c|c|c|c|c|c|}
\hline \multirow[b]{2}{*}{ Symptom or medication } & \multicolumn{3}{|c|}{ Univariate analysis } & \multicolumn{3}{|c|}{ Multivariate analysis } \\
\hline & OR & $95 \% \mathrm{CI}$ & P-value & OR & $95 \% \mathrm{CI}$ & P-value \\
\hline Heart failure & 5.078 & $1.973-13.067$ & $<0.001^{\mathrm{a}}$ & 1.846 & $1.080-2.611$ & $0.039^{\mathrm{b}}$ \\
\hline Hemoglobin $(<110 \mathrm{~g} / \mathrm{l})$ & 3.083 & $1.240-7.663$ & $0.011^{\mathrm{a}}$ & 3.599 & $2.078-5.119$ & $0.003^{\mathrm{b}}$ \\
\hline Baseline serum creatinine $(>106 \mu \mathrm{mol} / \mathrm{l})$ & 5.070 & $2.067-12.436$ & $<0.001^{\mathrm{a}}$ & 1.266 & $0.596-1.935$ & 0.656 \\
\hline eGFR (<60 ml/min) & 2.797 & $1.069-7.316$ & $0.029^{\mathrm{a}}$ & 1.598 & $0.260-2.937$ & 0.098 \\
\hline Aspirin 300 mg + clopidogrel & 6.794 & 2.812-16.419 & $<0.001^{\mathrm{a}}$ & 5.692 & $1.962-9.421$ & $<0.001^{\mathrm{b}}$ \\
\hline Diuretics & 2.625 & $1.206-5.715$ & $0.012^{\mathrm{a}}$ & 1.690 & $1.307-2.072$ & 0.226 \\
\hline
\end{tabular}

${ }^{a} \mathrm{P}<0.05$ in the univariate analysis; ${ }^{\mathrm{b}} \mathrm{P}<0.05$ in the multivariate analysis. DKI, delayed kidney injury; CI, confidence interval; OR, odds ratio; eGFR, estimated glomerular filtration rate.

order to examine this hypothesis, 436 cases that underwent CAG were enrolled. The results demonstrated that $7.1 \%$ patients (31/436) developed DKI according to the agreed diagnostic criteria. Thus, DKI existed in patients after CAG. DKIs occurring 1 month or later after coronary angiography had not been previously reported. All of the enrolled cases had already excluded CIN, therefore the renal toxicity of the contrast may not be able to account for the kidney injury within 1-6 months after CAG. Two questions remain, including whether DKI is associated with CAG or coronary heart diseases; and what pathogenesis underlies DKI. In the present study, the possible risk factors were assessed.

The Mehran score is a useful tool for predicting CIN risk, indicating the anti-stress capability of the kidney $(7,14)$. Therefore, the Mehran score was introduced to assess the risks for DKI in the present study. Through stratification by the Mehran score, it was revealed that the incidence of DKI increased with the Mehran risk scores. In the present cohort, $22.5 \%$ of patients with Mehran scores $\geq 16$ were diagnosed with DKI. The univariate analysis revealed that the serum levels of creatinine in the DKI group were higher than those in the non-DKI group, while $\mathrm{Hb}$ and eGFR levels were lower. Patients with DKI were characterized as older, and with a higher incidence of heart failure and cerebrovascular diseases. The analysis of oral drugs after CAG during the follow-up demonstrated that aspirin was used more frequently in the DKI group. The multivariate logistic regression analysis suggested that anemia, heart failure and $300 \mathrm{mg}$ aspirin intake were risk factors for DKI, while eGFR, diabetes, hypertension, ACE inhibitors/ARBs and statins were not.

Numerous drugs are used in patients that have undergone coronary stenting in order to cure several combined disorders; these drugs include anti-hypertensive drugs, statins and anti-platelet drugs. It is established that several hypotensors, statins and anti-platelet drugs cause renal toxicity, particularly in aged patients with declined renal function. There are several anti-platelet treatments used to prevent stent thrombosis and reduce future ischemic events, aspirin being one important and common anti-platelet therapy (16). As one of the traditional non-steroid, anti-inflammatory drugs, aspirin intake may result in renal failure or aggravate renal dysfunction $(17,18)$. In addition, the use of anti-platelets is a routine therapy in the treatment of CKD in order to prevent the recurrence of stroke or cardiovascular complications, since CKD increases the risk of incident stroke, heart failure and myocardial infarction (19).

Furthermore, heart failure is important in the decline of renal function in CKD patients, which often leads to death or other complications, such as pulmonary infection (20-23). Nowadays, it is considered that CIN is a short-term syndrome based on the pathophysiology, laboratory research and clinical studies (24-27). It is currently accepted that only a creatinine level increase within 7 days after CAG should be considered CIN (6). Thus, contrast-induced delayed kidney injury is actually not caused by contrast agents directly. Furthermore, the data of the present study demonstrated that $300 \mathrm{mg}$ aspirin and heart failure may account for DKI after CAG in a Chinese population. Finally, it is speculated that DKI will attract increasingly more attention in future studies. As a result, it is recommended that using large doses of aspirin as a long-term anti-platelet therapy be avoided in Chinese patients, and for high-risk patients, the long-term renal function follow-up results should not be ignored.

In conclusion, delayed CIN after CAG is not a logical term, while delayed kidney injury after CAG appears to be more appropriate when used to describe the renal injury occurring 1 month after CAG. The data of the present study suggested that $300 \mathrm{mg}$ aspirin and deteriorated heart function may contribute to the pathogenesis of DKI following CAG, and that iodinated contrast media is not a factor. However, the small cohort size of the current study is a limiting factor, and for this reason a follow-up study with a larger cohort is required in order to investigate delayed renal injury after $\mathrm{CAG}$, in addition to a subsequent prospective study in order to understand the etiology of DKI after CAG.

\section{Acknowledgements}

The current study was sponsored by the National Natural Science Foundation of China (grant no. 81570603), the New-100 Talent Plan of Shanghai Jiao Tong University School of Medicine (grant no. 2012) and the Shanghai Talents Development Fund (grant no. 2013). We would also like to thank Dr Gary C. Mouradian Jr. from the Medical College of Wisconsin for his English editing of the manuscript. 


\section{References}

1. Koo HM, Doh FM, Ko KI, Kim CH, Lee MJ, Oh HJ, Han SH, Kim BS, Yoo TH, Kang SW and Choi KH: Diastolic dysfunction is associated with an increased risk of contrast-induced nephropathy: A retrospective cohort study. BMC Nephrol 14: 146, 2013.

2. James MT, Ghali WA, Tonelli M, Faris P, Knudtson ML, Pannu N, Klarenbach SW, Manns BJ and Hemmelgarn BR: Acute kidney injury following coronary angiography is associated with a long-term decline in kidney function. Kidney Int 78: 803-809, 2010.

3. Kelly AM, Dwamena B, Cronin P, Bernstein SJ and Carlos RC: Meta-analysis: Effectiveness of drugs for preventing contrast-induced nephropathy. Ann Intern Med 148: 284-294, 2008.

4. McCullough PA: Contrast-induced acute kidney injury. J Am Coll Cardiol 51: 1419-1428, 2008.

5. World Medical Association: World Medical Association Declaration of Helsinki: Ethical principles for medical research involving human subjects. J Postgrad Med 48: 206-208, 2002.

6. Solomon R: Contrast-induced acute kidney injury (CIAKI). Radiol Clin North Am 47: 783-788, 2009.

7. Mehran R, Aymong ED, Nikolsky E, Lasic Z, Iakovou I, Fahy M, Mintz GS, Lansky AJ, Moses JW, Stone GW, et al: A simple risk score for prediction of contrast-induced nephropathy after percutaneous coronary intervention: development and initial validation. J Am Coll Cardiol 44 1393-1399, 2004.

8. Gault MH, Longerich LL, Harnett JD and Wesolowski C: Predicting glomerular function from adjusted serum creatinine. Nephron 62: 249-256, 1992.

9. Toprak O: Conflicting and new risk factors for contrast induced nephropathy. J Urol 178: 2277-2283, 2007.

10. Davidson C, Stacul F, McCullough PA, Tumlin J, Adam A, Lameire $\mathrm{N}$ and Becker CR; CIN Consensus Working Panel: Contrast medium use. Am J Cardiol 98: 42K-58K, 2006.

11. Reed M, Meier P, Tamhane UU, Welch KB, Moscucci M and Gurm HS: The relative renal safety of iodixanol compared with low-osmolar contrast media: A meta-analysis of randomized controlled trials. JACC Cardiovasc Interv 2: 645-654, 2009.

12. Mueller C, Buerkle G, Buettner HJ, Petersen J, Perruchoud AP, Eriksson U, Marsch S and Roskamm H: Prevention of contrast media-associated nephropathy: Randomized comparison of 2 hydration regimens in 1620 patients undergoing coronary angioplasty. Arch Intern Med 162: 329-336, 2002.
13. Hogan SE, L'Allier P, Chetcuti S, Grossman PM, Nallamothu BK Duvernoy C, Bates E, Moscucci M and Gurm HS: Current role of sodium bicarbonate-based preprocedural hydration for the prevention of contrast-induced acute kidney injury: A meta-analysis. Am Heart J 156: 414-421, 2008.

14. Mehran R and Nikolsky E: Contrast-induced nephropathy: Definition, epidemiology and patients at risk. Kidney Int Suppl: S11-S15, 2006.

15. Morcos SK: Prevention of contrast media nephrotoxicity - the story so far. Clin Radiol 59: 381-389, 2004.

16. Tanaka A, Ishii H, Sakakibara M, Okumura S, Jinno Y, Okada K, Suzuki S, Inoue Y and Murohara T: Temporary adjunctive cilostazol vs. clopidogrel loading for ST-segment elevation acute myocardial infarction. Am J Cardiovasc Drugs 14: 131-136, 2014.

17. Kim SJ and Bang OY: Antiplatelet therapy for preventing stroke in patients with chronic kidney disease. Contrib Nephrol 179: 119-129, 2013.

18. Harirforoosh S, Asghar W and Jamali F: Adverse effects of nonsteroidal antiinflammatory drugs: An update of gastrointestinal, cardiovascular and renal complications. J Pharm Pharm Sci 16: 821-847, 2013

19. Harmon JP, Zimmerman DL and Zimmerman DL: Anticoagulant and antiplatelet therapy in patients with chronic kidney disease: Risks versus benefits review. Curr Opin Nephrol Hypertens 22: 624-628, 2013.

20. Braam B, Joles JA, Danishwar AH and Gaillard CA: Cardiorenal syndrome-current understanding and future perspectives. Nat Rev Nephrol 10: 48-55, 2014.

21. Shamseddin MK and Parfrey PS: Mechanisms of the cardiorenal syndromes. Nat Rev Nephrol 5: 641-649, 2009.

22. Anand IS: Cardiorenal syndrome: A cardiologist's perspective of pathophysiology. Clin J Am Soc Nephrol 8: 1800-1807, 2013.

23. Clementi A, Virzi GM, Goh CY, Cruz DN, Granata A, Vescovo G and Ronco C: Cardiorenal syndrome type 4: A review. Cardiorenal Med 3: 63-70, 2013.

24. Haller $\mathrm{C}$ and Hizoh I: The cytotoxicity of iodinated radiocontrast agents on renal cells in vitro. Invest Radiol 39: 149-154, 2004.

25. Heyman SN, Reichman J and Brezis M: Pathophysiology of radiocontrast nephropathy: A role for medullary hypoxia. Invest Radiol 34: 685-691, 1999.

26. Liss P, Nygren A, Erikson U and Ulfendahl HR: Injection of low and iso-osmolar contrast medium decreases oxygen tension in the renal medulla. Kidney Int 53: 698-702, 1998.

27. Deek H, Newton P, Sheerin N, Noureddine S and Davidson PM: Contrast media induced nephropathy: A literature review of the available evidence and recommendations for practice. Aust Crit Care 27: 166-171, 2014. 